\title{
Interviewer Effects among Older Respondents in the European Social Survey
}

\begin{abstract}
The proportion of elderly people in general population samples is increasing. Therefore, it is becoming more important to pay special attention to older respondents when assessing the quality of data. The main hypothesis of the current paper is that interviewer effects are higher in the older age group. We use data collected in 13 countries during round 7 of the European Social Survey (ESS). The results support the supposition that older respondents tend to need more clarification, are more prone to misunderstand the questions, and are likely to have longer interviews. In line with the expectations, we also observe that among older respondents, particularly those aged 71 and above, interviewer effects are more common than among younger respondents.
\end{abstract}

Keywords: interviewer variance, intra-class correlation coefficient, interviewer-respondent interaction, survey data quality, elderly respondents

Interviewer Effects among Older Respondents in the European Social Survey 
The proportion of elderly people is increasing in the European population, and according to Eurostat (2015), the composition of Europe's population in terms of age is also changing. The percentage of people aged 65 and above was about $18.5 \%$ in 2014 , and is predicted to grow to $28.5 \%$ by 2050. In the European Social Survey (ESS) datasets (available at http://www.europeansocialsurvey.org/data), it can similarly be observed that the average age of respondents increased by 2.4 years from round 1 (2002-2003) to round 7 (2014-2015). The proportion of elderly people (aged 65 and above) increased by about 4 percentage points during the same period, and in round 7 of the ESS, about $24 \%$ of the respondents were 65 or older.

The growing segment of elderly people may have political, social, and commercial impacts on society, therefore, understanding how best to capture their opinions, perceptions, and beliefs will be of increasing interest to survey researchers. However, older adults are also considered a unique population with regard to survey methodology. Not only is it expected that their cognitive functioning and physical health will not be as good as those of the general population, it is also expected that their adoption of new ways of communication and technology will be different (Quinn, 2010). However, because the ESS is administered in a face-to-face mode, the upcoming challenges regarding the interface of technology and data collection as it relates to aging populations are not considered here.

During the various stages of a survey life cycle, many impediments need to be taken into account, specifically regarding older people. First, the sampling frame may become outdated more quickly for older than for younger people, because of higher mortality and morbidity rates among the elderly. Second, as older adults are more likely to live in institutions (e.g., care homes), sampling might become more complicated if the frames are address or household based. In the ESS, the population is demarcated as "all persons aged 15 and over [no upper age limit] resident within private households in each country, regardless of their nationality, citizenship or language," which suggests that people living in institutions are not eligible. As a result of these issues, the proportion of elderly people in the sample could be lower than the proportion of elderly people in 
the country as a whole. Third, age might also be a factor that determines unit (non)response. However, for the ESS round 7, the evidence is far from conclusive. In Belgium, Denmark, Germany, Slovenia, and Spain, unit nonresponse was slightly higher among the elderly, whereas in Estonia, Finland, Norway, Poland, Sweden, and Switzerland, the elderly instead were overrepresented (Beullens, Loosveldt, Vandenplas \& Denies, 2016).

Notwithstanding these important issues concerning selection, the focus in this paper is on measurement issues related to older respondents. Properly answering survey questions requires the respondent to understand the question, then retrieve the relevant information from memory, process that information, and lastly correctly format the answer in the response scale (Tourangeau, 1984). All the basic cognitive competencies that are needed during the process of providing an answer to the survey question can relate to age. In this regard, Hartshorne and Germine (2015) found thatcognitive peak performance is related to age. Their findings suggest that cognitive abilities related to vocabulary peaks around the age of 65 , working memory excels around the age of 30, and processing speed is highest around the age of 20.

According to Roedder and Cole (1986), cognitive and communicative functioning depends on the question difficulty (indicated by the amount of information presented to the respondent), and the format of the questions and possible answers. This specifically relates to information processing deficits in older people. Thus, particularly difficult questions pose more memory capacity problems and may lead to incomplete or biased responses, or to item nonresponse (Schwarz, Park, Knäuper \& Sudman, 1995; Schwarz \& Knäuper, 2000; Schwarz, 2005). According to the findings of Fox, Sidani, and Streiner (2007), older adults seem to struggle more with closed-ended questions that have numeric response formats. Questions that use a bidirectional scale (e.g., a four-point scale ranging from 1: "yes, that is true" to 4: "no, that is not true") in particular may be more cognitively demanding. Fox and colleagues (2007) also found evidence that older respondents prefer to express their answers in a verbal manner (open-ended format), instead of by selecting numbers. The interviewers in the research by Krestar, Looman, Powers, Dawson, and Judge (2012) observed that memory-impaired older adults tend instead to give 
yes/no answers to four point scales; particularly for bidirectional scales (as opposed to unidirectional). It is therefore advisable to use unidirectional scales instead of bidirectional, in order to reduce the cognitive burden for (older) respondents.

Older people can also have reduced capacity for memory retrieval, which can in turn lead to suboptimal answering strategies or satisficing behavior (Herzog \& Rodgers, 1992; Craik, 1999). For example, older respondents might have difficulty in remembering fragments of a question or possible answers after the interviewer has read the entire question and the alternative answers. Moreover, retrieving information from past events may be more difficult for older respondents (Craik, 1999). This can also involve erroneous recall or recognition (Schacter, Koutstaal, \& Norman, 1997). Research by Ziniel (2008) suggests that older respondents tend to use different memory strategies to answer behavioral frequency questions and use less episodic enumeration. Short-term memory issues may emerge during an interview if respondents are required to hold, process, and evaluate information for one question and its alternative answers (Craik, 1999; Salthouse \& Babcock, 1991). Knäuper and colleagues (2016, p. 186) concluded that "age-related changes in cognitive and communicative functioning can lead to age-related differences in selfreports that are erroneously interpreted as real age differences in attitudes and behaviors."

In addition to declining abilities among older adults, Gove and Geerken (1977) and Rodgers and Herzog (1977) also reported that older respondents tend to be more reluctant to provide embarrassing information and to produce higher scores for socially desirable items. In addition, more “don't know" answers are found to be given by older adults (Colsher \& Wallace, 1989).

All these considerations suggest that, in general, older adults cope differently with a survey interview than younger adults. This can also affect the interaction between the respondent and the interviewer. In this regard, van der Zouwen, Smit, and van der Horst (2005) found that interviewers tend to separate survey questions into smaller sub-questions for older respondents, as a means to compensate for question difficulty. Such deviations from the original questionnaire script lead to less rounding off and less overestimation of items, such as the average time spent on 
household tasks. However, this latter variable is more prone to bias, as these self-reports deviate more from recorded diary outcomes.

Overall, based on the presented results it can be hypothesized that interviewers play a more active role when dealing with older people, which is why increased interviewer variance can be observed among older respondents. This issue is particularly relevant for the ESS, in which interviewer variance is observed frequently, particularly in certain participating countries such as Bulgaria, Cyprus, the Czech Republic, Lithuania, the Russian Federation, and Ukraine (Beullens \& Loosveldt, 2016). Groves and Magilavy (1980, 1986) hypothesized increased interviewer variance among older respondents, and they offered some evidence from telephone surveys suggesting that older respondents tend to have higher intra-interviewer correlations. However, surprisingly, respondents in the age group from 35 to 49 also show relatively high intrainterviewer correlations. Earlier work on interviewer variance by Freeman and Butler (1976) also illustrates the impact of age on interviewer variance. They reported that "Old interviewers interviewing 'old' respondents produced consistently more interviewer variance than any other age combination" (p. 88).

In the current paper, we test whether interviewing older adults leads to increased interviewerrespondent interaction, which is indicated by longer interviews and interviewers reporting more respondent role issues during the interview, such as asking for clarification and not understanding the questions. More specifically, it can be expected that interviewers will report greater need for clarification among interviews with older respondents, and that these respondents will tend to be less likely to fully understand the questions. The intensified interviewer-respondent conversational interaction that might result from this could increase the likelihood of observing higher interviewer variance among older respondents. It is clear that the evaluation of higher interviewer variance among older respondents implies a comparison with other age groups, and that as a consequence, the age of respondents will be the key variable in our analysis. We also investigate whether the substantive topic and the response scales of the questions influence the expected higher interviewer effects among the elderly. 


\section{Data}

The ESS is a biennial, cross-national, and cross-sectional survey, conducted in 36 European countries. The first round was fielded in 2002 and fieldwork for round 7 started in 2014. Only round 7 is used for our analysis, and only the countries for which primary sample unit (PSU) information is available are included. These are Austria, Belgium, Czech Republic, Germany, Spain, France, the United Kingdom, Hungary, Ireland, Lithuania, Poland, Portugal, and Slovenia. In these countries, a total of 25,305 respondents were interviewed by 1,567 interviewers.

Respondents' age, with a special focus on the oldest age groups, is the main variable of interest in this paper, as it is expected to relate to the respondent-interviewer interaction and to interviewer effects. It is not clear in advance whether this relationship is strictly linear; instead, a curve-linear relationship could also be anticipated. Hence, in order to allow some flexibility in the analyses, age is recoded into seven categories: 26 or younger $(14.8 \%), 27$ to $36(14.8 \%), 37$ to $45(14.3 \%)$, 46 to $53(13.6 \%), 54$ to $62(15.2 \%), 63$ to $70(13.0 \%)$, and 71 or older $(14.2 \%)$. The percentages in parentheses indicate the relative size of each age group, aggregated over all countries of round 7 of the ESS. The intervals of the age groups were chosen so that the age groups are fairly equal in size.

The topics in the ESS cover a wide range of societal issues such as health, religion, work, education, ethnic background, and attitudes toward migration, politics, and justice. In order to measure interviewer effects (intra-class correlations), we selected a set of 72 questions related to health, attitudes towards societal issues, media use, and so forth. We determined the intra-class correlation for each of these variables, within each country and age group (see also the methods section). A list of the items can be found in the appendix. All the variables were treated as continuous. We opted for a heterogeneous set of both factual and attitudinal questions about different substantive topics with different response scales. Such a mix of questions is representative of a normal questionnaire, and therefore the results illustrate what could be 
expected in ordinary survey practice. The mix of questions also allows us to examine which variables are most sensitive to the higher interviewer effects expected to be found among older respondents. In this context, we considered both the substantive aspect of the questions and the response scales used.

In addition to the age variable and the 72 survey questions, we selected five additional variables in order to indirectly assess the interaction between interviewers and respondents during interviews in the different age groups. Four of these variables are interviewer assessments of the respondent, which needed to be filled out by the interviewer after the interview. These questions are:

- $\quad$ (RESCLQ) Did the respondent ask for clarification of any questions?

- $\quad($ RESBAB) Did you feel that the respondent tried to answer the questions to the best of his or her ability?

- (RESRELQ) Did you feel that the respondent was reluctant to answer any questions?

- (RESUNDQ) Overall, did you feel that the respondent understood the questions?

All four questions were answered on a 5-point scale $(1=$ never; $2=$ almost never; $3=$ now and then; 4 = often; 5 = very often). The fifth variable indicative of the respondent-interviewer interaction is the interview length (duration). The length of interviews is expected to vary between age groups, with older respondents having the longest durations. Throughout the course of the consecutive rounds of the ESS, many countries changed their interviewing technology to computer-assisted personal interviewing (CAPI). However, in the ESS round 7, Lithuania, Poland, and Spain still used paper and pen methods, which somewhat complicates the recording of the interview time. Interview durations are not available for Portugal in round 7. In some cases, the duration of the interview-measured as the difference between the start and end time recorded by the interviewer-shows unrealistic values (e.g., more than 200 minutes, less than 20 minutes, or even negative numbers) (Loosveldt \& Beullens, 2013). These unrealistic values are treated as missing values. 


\section{Methods}

The main hypothesis, that interviewer variance will be higher among older respondents, was tested in two steps. First, intra-class correlations (ICCs) were estimated for each of the selected questions (72) in different age groups (7) and countries (13). This resulted in a dataset with 6,552 ICCs $(72 \times 7 \times 13)$ and contextual information for each ICC. The contextual information consists of the identification of the question, age group, and country. In the second step, these ICCs were considered as the dependent variable to be modeled by the same set of variables (age groups, countries, and survey questions). It was particularly expected that increased ICCs would be observed for the older age groups.

Interviewer variance in the first step was measured by the intra-class correlation, modeled as

$$
\boldsymbol{I C C}=\frac{\sigma_{i n t}^{2}}{\sigma_{i n t}^{2}+\sigma_{p s u}^{2}+\sigma_{\varepsilon}^{2}}
$$

where $\sigma_{\text {int }}^{2}$ is the variance of a variable attributable to the interviewer level, $\sigma_{\text {psu }}^{2}$ represents the variance attributable to the areas or PSUs (primary sample units), and $\sigma_{s}^{2}$ is the residual variance. These variance components were assumed to be normally distributed and mutually independent. These terms can usually be obtained from multilevel models showing a hierarchical data structure where respondents are nested within interviewers and PSUs (Hox, 1994). Interviewers and PSUs were cross-classified.

If the PSUs are not included, the ICCs might not only indicate the differences between interviewers, but might also express area differences (O’Muircheartaigh \& Campanelli, 1998; Schnell \& Kreuter, 2005), as interviewers are usually assigned to sample units who live in the same region or area as the interviewer. The interpenetration of interviewers and areas is an important condition in order to separate these effects (Mahalanobis, 1946; Biemer \& Stokes, 1985). Ideally, interviewers are assigned (nationwide) to several local areas, which are in turn also assigned to other interviewers. In large-scale surveys, such conditions are very hard to realize. Therefore, the interpenetration is only realized locally, with more than one interviewer dealing 
with one PSU and vice versa. However, including the PSUs in the multilevel model allowed us to separate the interviewer and area variance, and to avoid confounding effects and thereby erroneously attributing area effects to the interviewer level.

The higher the ICC, the more interviewers tend to add 'noise' to the measurement of the survey variable, obviously decreasing its quality. The ICC is also an indication of the extent to which the answers of respondents are clustered within the interviewer to whom they are assigned, and is considered as an indication of the extent to which the interviewer plays a role in the process during which the respondent forms answers.

In this paper, the ICCs for the 72 questions were obtained separately for the combination of 7 age groups and 13 countries. As already mentioned, 6,552 ICCs were calculated. Subsequently, the contextual information was used to model these ICCs by the three independent variables: survey question, age group, and country.

Once all the ICCs had been obtained, they could be modeled, meaning that the ICCs were used as the dependent variable in the model. This is the second step in our procedure. The following multilevel model was used to assess how the ICCs arerelated to the age, country, and the survey questions:

$$
\operatorname{ICC}_{\text {aqc }}=\beta_{0 q c}+\sum_{a=2}^{7} \beta_{a} \text { Ageclass }_{a}+\varepsilon_{\text {aqc }}
$$

where

$$
\beta_{0 q c}=\gamma_{000}+\mu_{0 q 0}+v_{00 c}
$$

In total, we calculated 6,552 ICCs, meaning that there are 6,552 units in our analysis. Index $a$ identifies the age group $(a=1,2, \ldots, 7)$, index $q$ identifies the question $(q=1,2, \cdots, 72)$, and index $c$ identifies the country $(c=1,2, \cdots, 13)$. Accordingly, $I C C_{a q c}$ is the value of an ICC calculated for question $q$ in age group $a$ and country $c$. The combination of the indices $a$ and $c$ identify the groups in which the ICCs for each question $q$ were calculated. This means that 91 (7*13) ICCs were calculated for each question. So the ICCs are nested within questions and can 
vary over questions. For each country, we obtained $504(72 * 7)$ ICCs; the ICCs are also nested within countries and can vary over countries. To take into account the differences between questions and countries, country and question were included in the model as random effects, rendering a cross-classified multilevel model (Hox, 2010; Raudenbush \& Bryk, 2002; Snijders \& Bosker, 1999). This means that the random intercept in the model with ICC as the dependent variable can vary for the countries and the questions, implying that one variance parameter will be obtained for the effect of country and one variance parameter for the effect of the survey question, instead of a set of fixed parameters per country and per survey question. Age is a categorical variable with age class 14-26 as the reference group. In the model, age is specified as a fixed effect. This means that we obtained a regression coefficient for each age group.

The intercept structure of Model $2\left(\beta_{0 q \sigma}\right)$ includes a general intercept $\left(\gamma_{000}\right)$ that expresses the expected ICC for age class 14-26 (reference group), for a random country and survey question. The random intercepts $\mu_{0 q 0}$ (question-specific part of the intercept) allow the different survey questions to deviate from this general intercept, as does $v_{00 c}$ (country-specific part of the intercept) for the countries. The variances $\sigma_{\mu_{0}}^{2}$ and $\sigma_{v_{0}}^{2}$ express the magnitude of these deviationsfrom the general intercept for survey questions and countries, respectively.

The random intercepts concerning the questions $\mu_{0 q 0}$ and those concerning the countries $v_{0 q \mathrm{q}}$ are assumed to be normally distributed with mean zero and variances respectively termed $\sigma_{\mu_{0}}^{2}$ and $\sigma_{v_{0}}^{2}$. The term $\sum_{a=2}^{7} \beta_{a}$ Ageclass $_{a}$ in Model 2 is the essential set of parameters to be tested. The older age groups in particular are expected to show higher ICCs.

The ICCs were multiplied by 100 , in order to obtain interpretable parameters, and the model will also converge more easily this way. One last important issue needs to be mentioned: ICCs are generally expected to be non-negative, as they essentially express a variance. Nevertheless, negative variances are still possible, and when many ICCs are estimated, the occurrence of negative ICCs is relatively likely. Many statistical software programs censor 
negative variances at 0 , which might bias the results upward. Therefore, the mixed procedure in the SAS software was used, allowing negative ICC values.

\section{Results}

First, we present some descriptive results, assessing the conversational interaction between interviewers and respondents that is expected to be more elaborated among older respondents. Thereafter, the analysis is presented as to whether interviewer effects are indeed observed more among older respondents.

The results from Table 1 are strongly suggestive of a more intensive interviewerrespondent interaction among older respondents. On a 1 to 5 scale, the oldest age group (71 and above) is on average 0.55 scale points higher than the younger age groups with regard to the need for clarification (RESCLQ): the four youngest age groups need clarification "almost never," but this is close to "every now and then" for the 71 and above group. In addition, for the understanding of the questions (RESUNDQ) and the respondents' reluctance to provide answers (RESRELQ), the means for the older age groups tend to indicate a less favorable interview context. A similar tendency can be observed for motivation (RESBAB), although the difference is generally relatively modest compared with the other three variables concerning the interviewers' assessment of response behavior. For the four variables, the age effect follows a somewhat J-shaped curve. Roughly, up to the 54-62 age group, little difference can be found among the respondents. It is only in the two oldest age groups that the differences become very clear, with the oldest age group (71 and above) being the most distinct. The shape of the increasing curve regarding the total duration of the interview is somewhat different, with older respondents tending to need longer interviews, and the youngest age group having the shortest interview times. This also suggests more extensive and conversational interaction among older respondents.

[Table 1 about here.] 
The results in Table 1 strongly suggest that older respondents may need to rely much more on the assistance of the interviewer, as indicated by their greater need for clarification, less understanding of the question, more reluctance, and longer interviews. As a result, it seems reasonable to hypothesize that older respondents are also more prone to be influenced by interviewers.

With regard to the main research question, the last column in Table 1 provides the mean ICCs per age group. Generally, it is striking that ICCs of 0.10 and more are observed in the ESS, which suggests that a more detailed understanding of this phenomenon is needed. The column also shows that the impact of the interviewers is greatest among the eldest respondents. Whereas the average ICCs are about 0.10 to 0.12 among the age groups up to 63-70, the ICC for the age group 70 and above is 0.14 . These first results seem to suggest a J-shaped curve effect of age on ICCs.

More information about the ICCs for the age group 70 and above is presented in the Appendix with the 72 questions. The last column of the appendix contains the ICCs for each question separately, and the questions are ordered based on decreasing ICCs. The range of the ICCs is considerable [0.27-0.02]. Three quarters of the questions have an ICC value of more than 0.10. The list in the appendix also contains information about the substantive topic and the number of response categories $(4,5,7,11,>11)$. Two categories of substantive questions aredistinguished: personal questions (e.g., severe financial difficulties in the family when growing up) and questions about politics and society (e.g., laws against ethnic discrimination in the workplace). It is remarkable that many questions about politics and society are at the top of the list (high ICC values), whereas personal questions are closer to the bottom of the list. The pattern of the number of response categories is less obvious. The results of an analysis of variance with the ICC as the dependent variable, and the substantive topic and the number of response categories (categorical factor) as independent variables, supports this observation. There is no significant effect of the number of response categories $(\mathrm{F}=1.767 ; \mathrm{df}=4 ; \mathrm{p}>0.1)$. However, there is a significant effect of the substantive topic on the ICCs $(\mathrm{F}=39.072 ; \mathrm{df}=1 ; \mathrm{p}<0.001)$. For the age group 70 and above, the ICCs are significantly higher for the political and societal questions than for the personal questions. This seems to indicate that older people are more oriented toward their personal 
situation than on more general societal issues, making it more easy to answer personal questions than more general questions about politics and society. The interaction and the answering process for the personal questions probably runs more smoothly and requires less interviewer intervention, thereby reducing the risk of interviewer effects.

The results from the cross-classified model with countries and questions as random effects [i]

(Model 2) are shown in Table 2, and provide more details about the relationship between interviewer effects and respondents' age. The grand intercept $\left(\gamma_{000}=10.59\right)$ reflects the average ICC in round 7 of the ESS.

The important parameters of Model 2 concern the effects of age. The marginal model (fixed effect parameters $\beta_{2 q c}$ to $\beta_{7 q c}$ ) shows a relationship between age and the ICCs similar to that presented in Table 1, and confirms the J-shaped curve effect, as only the oldest age group (70 and above) is significantly different $(\mathrm{p}<0.001)$ from the reference group $(14-25)$. This significant difference supports the expectation of higher interviewer effects in the oldest age group.

[Table 2 about here.]

The differences in the overall levels of ICCs are much larger between countries than between survey questions. This can be observed from the random intercept variances: $80.03\left(\sigma_{v_{0}}^{2}\right)$ for countries and $12.50\left(\sigma_{\mu_{0}}^{2}\right)$ for survey questions. Figure 1 shows the relationship between age groups and average ICCs over the 72 survey questions for each of the 13 countries. Countries such as Austria, the Czech Republic, Hungary, Lithuania, and Ireland show high levels of interviewer effects in all age groups. The pattern where the oldest age group has the highest ICCs can clearly be observed in Belgium, France, Germany, Poland, Portugal, Slovenia, and Spain. These are all countries with lower or more moderate levels of interviewer effects. It could be hypothesized that in the high interviewer ICC countries, the interviewer effects are generated more by a mechanism of general interviewer satisficing (e.g., stimulating straightlining or other response patterns) that is active in each interview regardless of the response behavior and the type of respondent. On the 
other hand, in the low interviewer ICC countries, interviewer behavior seems influenced more by response behavior, and interviewer effects are instead a result of an intensified and more complex respondent-interviewer interaction. A supportive element in this regard is the fact that in the high interviewer ICC countries, the difference in interview duration between the youngest and oldest age category is only three minutes, whereas in the low interviewer ICC countries this difference is 17 minutes (with the older age group needing more time). Similarly, the mean age differences regarding the four respondent assessmentvariables (RESCLQ, RESRELQ, RESBAB, and RESUNDQ) are smaller in the high interviewer ICC countries compared with the low interviewer ICC countries, with the oldest age group showing less favorable respondent behavior (not reported in tables).

[Figure 1 about here.]

As already mentioned, the differences in ICCs between questions are less pronounced, and these differences also do not seem to be related to the topic or the response scale of the question. However, it can be noted that the expected pattern of higher interviewer effects in the oldest age group occurs in approximately half (35 out of 72 ) of the questions. The question characteristics that are related to this pattern are not clear and need further investigation. Possibly, more detailed characteristics about the complexity of the questions (e.g., the number of words), the answer categories (e.g., fully labeled or not), and the location of the question should be used (Yan\& Tourangeau, 2008)

\section{Conclusion and Discussion}

Given the fact that the group of older people is expanding, both in the population and in samples used for surveys, and is expected to be even larger in the future, the focus on elderly people in survey research is gaining importance. Previous research indicates that interviews with older respondents can proceed in a different way than interviews with younger respondents. The results presented in the current paper are in line with this observation. Older respondents need more 
interviewer assistance. This is supported by the findings that interviewers perceive older respondents to be less capable of understanding the questions, are more likely to ask for clarification, are somewhat more reluctant, and are somewhat less motivated. Further, interviews tend to take longer for older respondents. There are thus clear indications that the interviewerrespondent interaction is more intensive during interviews with older respondents. The assessment of interviewer effects by means of interviewer variance estimates in different age groups is a specific operationalization of this general idea. For that purpose, ICCs were calculated in 13 countries for 72 questions in 7 age groups. The PSUs were taken into account to separate interviewer and area effects. This procedure allowed us to specify the ICCs as dependent variables in a model with the age groups as independent variables. This approach implies an evaluation of the direct effect of age on the ICCs.

In line with expectations, we observed that among older respondents-particularly those aged 71 and above-interviewer effects are more common than among younger respondents, although this is not necessarily true for all countries and/or survey questions. Nevertheless, there is empirical support that interviews with older respondents are more complex, and result in higher ICCs. Accordingly, it is relevant to investigate data quality aspects for older respondents in greater detail (e.g., interviewer-respondent interaction, response styles such as non-differentiation and straightlining, and socially desirable answers). More specifically, it was found that interviewer effects are larger with regard to questionsabout political and social issues. These are topics for which the involvement of the elderly is probably smaller. The results also show that interviewer effects in general are more similar between survey questions than between countries.

Based on these results, a number of suggestions for further research and recommendations for practice can be made. With regard to further research, one could assess the impact of the higher interviewer effects for older respondents on the substantive analysis of the these variables. Due to the greater interviewer effects in the oldest age group, it can be expected that the estimates concerning substantive variables in this age group will be less precise. A second research suggestion would be to apply the method presented in the current paper to assess the impact on 
interviewer effects of other respondent characteristics. Age is probably not the only variable that is predictive of interviewer effects. The respondents' level of education or home language, for example, might also be important variables contributing to the involvement of the interviewer in the interview interaction, possibly also leading to differential interviewer effects. The model could also be elaborated, together with the analysis of interviewer characteristics. Related to research questions about interviewing elderly people, the interviewer's age is certainly eligible in this regard. The results also clearly indicate that it is worthwhile to investigate interviewer-related data quality aspects for older respondents in greater detail: interviewer-respondent interaction, the use of response styles such as non-differentiation and straightlining, and the tendency to give socially desirable answers.

In addition to these suggestions for future research, a number of recommendations can also be made for practice. These are related to the construction of questionnaires and to interviewer training. It could be suggested that the interviewer is the protagonist in creating interviewer effects. Therefore, there should be a focus on the way interviewers deal with respondent assistance or unfavorable respondent behavior. Respondent assistance provided by the interviewer to older (or less capable) respondents should therefore be more scripted or standardized, avoiding the possibility that respondent support becomes too interviewer specific. This could possibly be achieved by providing more intensive and adequate interviewer briefing and training. While designing the questionnaire, it must be ensured that the questions are sufficiently relevant for older age groups. The relevance and difficulty of the questions need to be evaluated from the perspective of the elderly. In addition, testing the questionnaire with a sufficiently large group of elderly people is essential.

To some extent, the interview process is a black box that should be revealed in much greater detail in order to better understand and remedy the issue of interviewer effects. More paradata such as interview recordings might help to better monitor interviewer behavior and possibly explain the origin of the observed country differences. 


\section{References}

Beullens, K., \& Loosveldt, G. (2016). Interviewer effects in the European Social Survey. Survey Research Methods, 10(2), 103-118. doi: http://dx.doi.org/10.18148/srm/2016.v10i2.6261

Beullens, K., Loosveldt, G., Vandenplas, C., \& Denies, K. (2016). Quality matrix for ESS R7 first and second release countries (Tech. Rep.). Leuven: KU Leuven, ESS-ERIC.

Biemer, P., \& Stokes, L. (1985). Optimal design of interviewer variance experiments in complex surveys. Journal of the American Statistical Association, 80, 158-166. doi: 10.2307/2288066

Colsher, P., \& Wallace, R. (1989). Data quality and age: Health and psychobehavioral correlates of item nonresponse and inconsistent responses. Journal of Gerontology, 44(2), 45-52. doi:10.1093/geronj/44.2.P45

Craik, F. (1999). Memory, aging and survey measurement. In N. Schwarz, D. Park, B. Knäuper, \& S. Sudman (Eds.), Cognition, aging and self-reports (pp. 95-115). Philadelphia: Taylor \& Francis.

Eurostat. (2015). People in the EU - population projection. Retrieved 21 August 2016 from http://ec.europa.eu/eurostat/statistics-explained/index.php/People_in_the_EU_\%E2\%80\% 93 population_projections

Fox, M., Sidani, S., \& Streiner, D. (2007). Using standardized survey items with older adults hospitalized for chronic illness. Research in Nursing \& Health, 30(4), 468-481. doi: 10.1002/nur.20201

Freeman, J., \& Butler, W. (1976) Some sources of interviewer variance in surveys. Public Opinion Quarterly, 40(1), 79-91. doi: 10.1086/268269

Gove, W., \& Geerken, M. (1977). Response bias in surveys of mental health. American Journal of Sociology, 82(6), 1289-1317. doi: 10.1086/226466 
Groves, R., \& Magilavy, L. (1980). Estimates of interviewer variance in telephone surveys. In Proceedings of the American Statistical Association, Section on Surrey Research Methods (pp. 622-627).

Groves, R., \& Magilavy, L. (1986). Measuring and explaining interviewer effects in centralized telephone surveys. Public Opinion Quarterly, 50(2), 251-266. doi: 10.1086/268979

Hartshorne, J., \& Germine, L. (2015). When does cognitive functioning peak? The asynchronous rise and fall of different cognitive abilities across the life span. Psychological Science, 26(4), 433-443. doi: 10.1177/0956797614567339

Herzog, A., \& Rodgers, W. (1992). The use of survey methods in research on older americans. In R. Wallace \& R. Woolson (Eds.), The Epidemiologic Study of the Elderly (pp. 60-90). Oxford: Oxford University Press.

Hox, J. (1994). Hierarchical regression models for interviewer and respondent effects. Sociological Methods and Research, 22(3), 300-318. doi: 10.1177/0049124194022003002

Hox, J. (2010). Multilevel analysis: techniques and applications (2nd ed.). New York: Routledge.

Knäuper, B., Carrière, K., Chamandy, M., Xu, Z., Schwarz, N., \& Rosen, N. (2016). How aging affects self-reports. European Journal of Ageing, 13, 185-193. doi: 10.1007/s10433-0160369-0

Krestar, M., Looman, W., Powers, S., Dawson, N., \& Judge, K. (2012). Including individuals with memory impairment in the research process: The importance of scales and response categories used in surveys. Journal of Empirical Research on Human Research Ethics: An International Journal, 7(2), 70-79. doi: 10.1525/jer.2012.7.2.70

Loosveldt, G., \& Beullens, K. (2013). How long will it take?' An analysis of interview length in the fifth round of the European Social Survey. Survey Research Methods, 7(2), 69-78. doi: $10.18148 / \mathrm{srm} / 2013 . \mathrm{v} 7 \mathrm{i} 2.5086$ 
Mahalanobis, P. (1946). Recent experiments in statistical sampling in the Indian Statistical Institute. Journal of the Royal Statistical Society, 109, 325-370.

O’Muircheartaigh, C., \& Campanelli, P. (1998). The relative impact of interviewer effects and sample design effects on survey precision. Journal of the Royal Statistical Society: Series A (Statistics in Society), 161(1), 63-77. doi: 10.111/1467-985X.00090

Quinn, K. (2010). Methodological considerations in surveys of older adults: Technology matters. International Journal of Emerging Technologies and Society, 8(2), 114-133.

Raudenbush, S., \& Bryk, A. (2002). Hierarchical linear models: Applications and data analysis methods. Thousand Oaks (California): Sage.

Rodgers, W., \& Herzog, A. (1987). Interviewing older adults: The accuracy of factual information. Journal of Gerontology, 42(4), 387-394. doi: 10.1093/geronj/42.4.387

Roedder John, D., \& Cole, C. (1986). Age differences in information processing: Understanding deficits in young and elderly consumers. Journal of Consumer Research, 13(3), 297-315. doi: $10.1086 / 209070$

Salthouse, T., \& Babcock, R. (1991). Decomposing adult age differences in working memory. Psychological Review, 27(5), 763-776. doi: 10.1037/0012-1649.27.5.763

Schacter, D., Koutstaal, W., \& Norman, K. (1997). False memories and aging. Cognitive Sciences, 1(6), 229-236. doi: 10.1016/S1364-6613(97)01068-1

Schnell, R., \& Kreuter, F. (2005). Separating interviewer and sampling-point effects. Journal of Official Statistics, 21(3), 389-410.

Schwarz, N. (2005). Aging and the psychology of self-report. In L. Carstensen (Eds.). When I'm 64: Aging frontiers in social psychology, and adult developmental psychology (pp. 219-230). Washington DC: National Academic Press. 
Schwarz, N., \& Knäuper, B. (2000). Cognition, aging and self-reports. In D. Park \& N. Schwarz (Eds.), Cognitive aging: A primer (pp. 233-252). Philadelphia: Psychology Press.

Schwarz, N., Park, D., Knäuper, B., \& Sudman, S. (1995). Cognition, aging, and self-reports. Philadelphia: Psychology Press.

Snijders, T., \& Bosker, R. (1999). Multilevel analysis: An introduction to basic and advanced Multilevel Modelling. London: Sage.

Tourangeau, R. (1984). Cognitive science and survey methods: A cognitive perspective. In T. Jabine, M. Straf, J. Tanur, \& R. Tourangeau (Eds.), Cognitive aspects of survey methodology: Building a bridge between disciplines. (pp. 70-100). Washington DC: National Academy Press.

van der Zouwen, J., Smit, J., \& van der Horst, M. (2005). Reporting the frequency and duration of household tasks by elderly respondents: The effect of different interview strategies on data quality. In Proceedings of the 60th AAPOR annual conference (pp. 4007-4014). Alexandria: American Statistical Association.

Yan, T., \& Tourangeau, R. (2008). Fast times and easy questions: The effect of age, experience and question complexity on web survey response time. Applied Cognitive Psychology, 21, 51 -68 Doi: 10.1002/acp.1331

Ziniel, S. (2008). Cognitive aging and survey measurement. (Unpublished doctoral dissertation). The University of Michigan. 
[i]

To test the sensitivity of Model 2, a model with country as the fixed effect and questions as random effects was tested. There were no differences in the results between the two models, except for the global intercept due to the selection of the reference category for the country variable (the reference category was Ireland, with a dummy variable for the other countries). 